\section{OPEN ACCESS}

Edited by:

Peter Hegyi,

University of Szeged, Hungary

Reviewed by:

Nandor Faluhelyi

University of Pécs Medical School,

Hungary

Shin Hamada,

Tohoku University, Japan

${ }^{*}$ Correspondence:

Stephen J. Pandol

Stephen.Pando@@cshs.org

Debiao $\mathrm{Li}$

Debiao.Li@cshs.org

${ }^{\dagger}$ These authors have contributed equally to this work

Specialty section:

This article was submitted to

Gastrointestinal Sciences,

a section of the journal

Frontiers in Physiology

Received: 12 July 2019

Accepted: 09 January 2020

Published: 21 February 2020

Citation:

Wang L, Gaddam S, Wang N, Xie Y, Deng Z, Zhou Z, Fan Z, Jiang T, Christodoulou AG, Han F, Lo SK, Wachsman AM, Hendifar AE,

Pandol SJ and Li D (2020) Multiparametric Mapping Magnetic Resonance Imaging of Pancreatic Disease. Front. Physiol. 11:8. doi: 10.3389/fphys.2020.00008

\title{
Multiparametric Mapping Magnetic Resonance Imaging of Pancreatic Disease
}

\begin{abstract}
Lixia Wang ${ }^{1 \dagger}$, Srinivas Gaddam ${ }^{2 \dagger}$, Nan Wang ${ }^{3,4}$, Yibin Xie ${ }^{4}$, Zixin Deng ${ }^{4}$, Zhengwei Zhou ${ }^{4}$, Zhaoyang Fan ${ }^{4}$, Tao Jiang ${ }^{1}$, Anthony G. Christodoulou 4 , Fei Han ${ }^{5}$, Simon K. Lo², Ashley M. Wachsman ${ }^{4}$, Andrew Eugene Hendifar ${ }^{6}$, Stephen J. Pandol2* and Debiao Li ${ }^{4 *}$

1 Department of Radiology, Beijing Chaoyang Hospital, Capital Medical University, Beijing, China, ${ }^{2}$ Division of Digestive and Liver Diseases, Cedars-Sinai Medical Center, Los Angeles, CA, United States, ${ }^{3}$ Department of Bioengineering, University of California, Los Angeles, Los Angeles, CA, United States, ${ }^{4}$ Cedars-Sinai Biomedical Imaging Research Institute, Los Angeles, CA, United States, ${ }^{5}$ Department of Nuclear Science and Engineering, Siemens Healthineers, Princeton, NJ, United States, ${ }^{6}$ Department of Gastrointestinal Malignancies, Cedars-Sinai Medical Center, Los Angeles, CA, United States
\end{abstract}

Background: Current magnetic resonance imaging (MRI) of pancreatic disease is qualitative in nature. Quantitative imaging offers several advantages, including increased reproducibility and sensitivity to detect mild or diffuse disease. The role of multiparametric mapping MRI in characterizing various tissue types in pancreatic disease such as chronic pancreatitis (CP) and pancreatic ductal adenocarcinoma (PDAC) has rarely been evaluated.

Purpose: To evaluate the feasibility of multiparametric mapping $[T 1, T 2$, and apparent diffusion coefficient (ADC)] in defining tissue characteristics that occur in CP and PDAC to improve disease diagnosis.

Materials and Methods: Pancreatic MRI was performed in 17 patients with PDAC undergoing therapy, 7 patients with CP, and 29 healthy volunteers with no pancreatic disease. T1 modified Look-Locker Inversion Recovery (T1 MOLLI), T2-prepared gradient-echo, and multi-slice single-shot echo-planar diffusion weighted imaging (SSEPI DWI) sequences were used for data acquisition. Regions of interest (ROls) of pancreas in PDAC, CP, and control subjects were outlined by an experienced radiologist. One-way analysis of variance (ANOVA) was used to compare the difference between groups and regions of the pancreas, and Tukey tests were used for multiple comparison testing within groups. Receiver operator characteristic (ROC) curves were analyzed, and the areas under the curves (AUCs) were calculated using single parameter and combined parameters, respectively.

Results: T1, T2, and ADC values of the entire pancreas among PDAC, CP, and control subjects; and between upstream and downstream portions of the pancreas in PDAC patients were all significantly different $(p<0.05)$. The AUC values were 0.90 for $T 1$, 0.55 for T2, and 0.71 for ADC for independent prediction of PDAC. By combining T1, T2, and ADC, the AUC value was 0.94 (sensitivity $91.54 \%$, specificity $85.81 \%, 95 \% \mathrm{Cl}$ : $0.92-0.96)$, which yielded higher accuracy than any one parameter only $(\rho<0.001)$. 
Conclusion: Multiparametric mapping MRI is feasible for the evaluation of the differences between PDAC, CP, and normal pancreas tissues. The combination of multiple parameters of $\mathrm{T} 1, \mathrm{~T} 2$, and $\mathrm{ADC}$ provides a higher accuracy than any single parameter alone in tissue characterization of the pancreas.

Keywords: pancreatic ductal adenocarcinoma, chronic pancreatitis, magnetic resonance imaging, parametric mapping, T1, T2, ADC

\section{INTRODUCTION}

Pancreatic cancer is the most common pancreatic malignant neoplasm and is the third most common cause of cancerrelated deaths from NCI data in 2018. In the United States, pancreatic cancer accounts for about $3 \%$ of all cancers in the United States and about $7 \%$ of all cancer deaths (American Cancer Society, 2018; Siegel et al., 2018). PDAC is the major subtype of exocrine tumor and constitutes more than $90 \%$ of all pancreatic malignancies. Because of the tumor's unique microenvironment and aggressive nature, PDAC has a relatively poor response to the conventional systemic chemotherapy and poor prognosis, with an overall 5-year survival rate of 8.5\% (Yamamoto et al., 2015). Studies found that CP has markedly increased risk for pancreatic cancer (Hao et al., 2017). Five years after diagnosis, CP has a nearly eightfold risk for pancreatic cancer (Kirkegard et al., 2017). Inflammation participates in the development of tumor initiation, progression, treatment response, metastasis, and prognosis (Shi and Xue, 2019). The typical histopathologic features of CP contain acinar cell atrophy, pancreatic fibrosis, leukocyte infiltration, fatty replacement, and distorted and blocked ducts. These findings in CP and PDAC suggest that there are similar radiologic appearances. Further, the upstream pancreas (toward the tail end of the tumor) can have changes of CP due to duct obstruction.

Contrast-enhanced CT was used in PDAC detection, staging, and evaluation of prognosis. A previous study also found that CT radiomics could predict PDAC SMAD4 status and tumor stromal content (Attiyeh et al., 2019). X-ray radiation and iodine allergy are the major risks of CT. Endoscopic ultrasonography (EUS) is the most sensitive non-operative imaging method for the detection of pancreatic cancer and showed to be superior to CT (Luz et al., 2014; Singh and Faulx, 2016). EUS-guided fine needle aspiration (FNB) can achieve cytological information. However, it is invasive and highly operator dependent.

Several studies aimed to quantitatively differentiate PDAC from CP or autoimmune pancreatitis using MR techniques (Park et al., 2009; Fukukura et al., 2012; Yin et al., 2017). DWI plays an important role in the identification of PDAC lesions from

Abbreviations: ADC, apparent diffusion coefficient; ANOVA, one-way analysis of variance; $\mathrm{AUC}$, area under curve; $\mathrm{COP}$, chronic obstructed pancreatitis; $\mathrm{CP}$, chronic pancreatitis; DWI, diffusion weighted imaging; MPD, main pancreatic duct; MRI, magnetic resonance imaging; PDAC, pancreatic ductal adenocarcinoma; ROC, receiver operator characteristic; T1 MOLLI, T1 modified Look-Locker Inversion Recovery; T1-VIBE-DIXON, T1-weighted volumetric interpolated breath-hold examination; T2 HASTE, T2 half-Fourier acquisition single-shot turbo spin-echo sequence; TACP, tumor-associated chronic pancreatitis. the background of pancreatic parenchyma (Ichikawa et al., 2007; Wang et al., 2011; Fukukura et al., 2012; Hayano et al., 2016). With high $b$-value DWI, PDAC lesions can be reliably detected as an increased focal hyper-intensity area (Fukukura et al., 2012).

Hecht et al. (2017) found that the ADC value was significantly lower in tumors with dense fibrosis and may serve as a biomarker of fibrosis architecture. Choi et al. (2016) found that DWI with ADC value was a promising method to differentiate PDAC from mass-forming autoimmune pancreatitis. However, the ADC value only reflects one aspect of the differences between PDAC and $\mathrm{CP}$ vs. the normal control pancreas.

$\mathrm{T} 1$ and $\mathrm{T} 2$ relaxation times are valuable as quantitative parameters to characterize different tissues, especially in myocardial and liver diseases (Apprich et al., 2012; Kali et al., 2015; Blystad et al., 2017; Chen et al., 2017; Vietti Violi et al., 2019). T1 mapping was shown to improve the diagnosis of myocarditis, infarction, iron overload, and amyloidosis (Guo et al., 2009; Karamitsos et al., 2013; Kali et al., 2015). Previous studies found that T1 mapping combined Gd-EOB-DTPAenhanced MRI can be used to predict the pathologic grading of hepatocellular carcinoma (Cieszanowski et al., 2012; Banerjee et al., 2014; Chen et al., 2017). Recently, Banerjee et al. (2014) and Cassinotto et al. (2015) found that T1 values are strongly correlated with liver fibrosis and liver biopsy in a population of 79 patients. Cieszanowski et al. (2012) demonstrated significantly higher sensitivity and accuracy of T2 relaxation times than ADC values (99.0 and $89.3 \%$ vs. 79.0 and $80.9 \%$, respectively) for diagnosing hepatic malignancy. Some studies focused on using the $\mathrm{T} 1$ value in the diagnosis and classification of $\mathrm{CP}$ and found that $\mathrm{T} 1$ could provide quantitative metrics for determining the presence and severity of acinar cell loss and aid in the diagnosis of CP (Tirkes et al., 2017, 2018; Wang et al., 2018). In an animal model of pancreatic cancer, Yin et al. (2017) found that multiparametric MRI was able to characterize pancreatic masses, suggesting that $\mathrm{T} 1, \mathrm{~T} 2$, and ADC mapping may have a direct clinical application in patients with PDAC.

In this study, we evaluate the feasibility of non-contrast multiparametric mapping (T1, T2, and ADC) in defining tissue characteristics that occur in CP and PDAC needed for advances in specific diagnosis. The pathological changes in these diseases are complex including changes in cellular density, blood supply, fibrosis, edema, and inflammation. Our pilot study was designed to determine if there are quantitative methods, which can provide specific biomarkers using our novel method non-contract MRI methods based on the hypothesis that a combination of quantitative measures of relaxation time ( $\mathrm{T} 1$ and $\mathrm{T} 2$ values) and $\mathrm{ADC}$ values will 
provide biomarkers that distinguish $\mathrm{PDAC}, \mathrm{CP}$, and normal control pancreas.

\section{MATERIALS AND METHODS}

\section{Study Population}

During the 18-month study period from October, 2017 to April, 2018, patients with PDAC, CP, and normal control pancreas were recruited into this study. All PDAC patients were confirmed by histopathology using tissue obtained by endoscopic ultrasonography (EUS)-guided FNA. The PDAC patients were undergoing neo-adjuvant chemotherapy at the time of the research MRI.

All the patients with $\mathrm{CP}$ had diagnosis established by magnetic resonance cholangiopancreatography (MRCP) or endoscopic retrograde cholangiopancreatography (ERCP) with Cambridge classification for CP (Freeny, 1989; Nattermann et al., 1993; Sahai et al., 1998; Tirkes et al., 2018).

The normal control group had no history of acute pancreatitis, $\mathrm{CP}$, diabetes, pancreatic surgery, and no family history of cancer. Patients with pancreatic cystic lesions, benign tumors, or marked pancreatic atrophy or fat degeneration on MRI images were excluded. This prospective study was approved by the local institutional review board. Written informed consents were obtained from all participants.

\section{Magnetic Resonance Imaging Technique}

All subjects were scanned on a 3.0T MR scanner (Biograph mMR, Siemens Healthcare Sector, Erlangen, Germany) with an 18-channel phase array surface coil and were placed headfirst, supine position in the magnet. Conventional qualitative sequences and non-contrast quantitative mapping sequences were run for each subject.
Conventional qualitative sequences were transversal T1-weighted three-dimensional volumetric interpolated breath-hold examination (VIBE) with Dixon fat saturation (T1-VIBE-DIXON), and T2 HASTE in transverse, coronal, and sagittal orientations. The parameters of T1-VIBE-DIXON are repetition time $(\mathrm{TR})=4.15 \mathrm{~ms}$; echo time $(\mathrm{TE})=1.39 / 2.65 \mathrm{~ms}$; flip angle $=9^{\circ}$; field of view $(\mathrm{FOV})=247 \times 330 \mathrm{~mm}$; acquisition matrix $=320 \times 180$; echo train length $(E T L)=2$; slice thickness $=3 \mathrm{~mm}$; iPAT acceleration factor $=3 . \mathrm{T} 2$ HASTE was performed with the following acquisition parameters: $\mathrm{TR}=1,000 \mathrm{~ms}$; $\mathrm{TE}=99 \mathrm{~ms}$; flip angle $=105^{\circ}$; FOV $=226 \times 330 \mathrm{~mm}$; matrix $=256 \times 176 ; \mathrm{ETL}=109$; slice thickness $=5 \mathrm{~mm}$; slice gap $=1 \mathrm{~mm}$; iPAT acceleration factor $=2$. MRCP was performed with the following parameters: $\mathrm{TR}=8,903 \mathrm{~ms}$; $\mathrm{TE}=701 \mathrm{~ms}$; flip angle $=100^{\circ}$; $\mathrm{FOV}=300 \times 300 \mathrm{~mm}$; acquisition matrix $=384 \times 384 ; \mathrm{ETL}=2$; slice thickness $=1 \mathrm{~mm}$; iPAT acceleration factor $=2$.

Non-contrast quantitative MRI sequences consisted of 2D MOLLI Trufi with motion correction for T1 mapping, 2D T2prepared FLASH for T2 mapping, and multi-slice single-shot echo-planar imaging (SS-EPI) for DWI and ADC mapping. T1 MOLLI was acquired using a three-point tool to localize the slice with a single 10-s breath-hold and the following parameters: acquisition window $=280.5 \mathrm{~ms} ; \mathrm{TE}=1.12 \mathrm{~ms}$; echo spacing $=2.7 \mathrm{~ms}$, simulated $\mathrm{R}-\mathrm{R}$ interval $=1,000 \mathrm{~ms}$; flip angle $=35^{\circ} ; \mathrm{FOV}=390 \times 390 \mathrm{~mm}$; matrix $=192 \times 144 ;$ iPAT acceleration factor $=2$. T2-prepared FLASH was acquired, and the slices of T1 MOLLI were copied with a single 10-s breath and the following parameters: acquisition window $=207.4 \mathrm{~ms}$; $\mathrm{TR}=3.15 \mathrm{~ms}, \mathrm{TE}=1.32 \mathrm{~ms}$; echo spacing $=3.1 \mathrm{~ms}$, simulated $\mathrm{R}-\mathrm{R}$ interval $=1,000 \mathrm{~ms}$, duration of $\mathrm{T} 2$ preparations $=0,30$, and $55 \mathrm{~ms}$; flip angle, $12^{\circ}$; FOV, $390 \times 390 \mathrm{~mm}$; matrix, $192 \times 144$; iPAT acceleration factor $=2$. For T1 MOLLI and T2-prepared FLASH, three slices located at, above, and below the tumor area

TABLE 1 | Parameters of all MRI sequences used in the study.

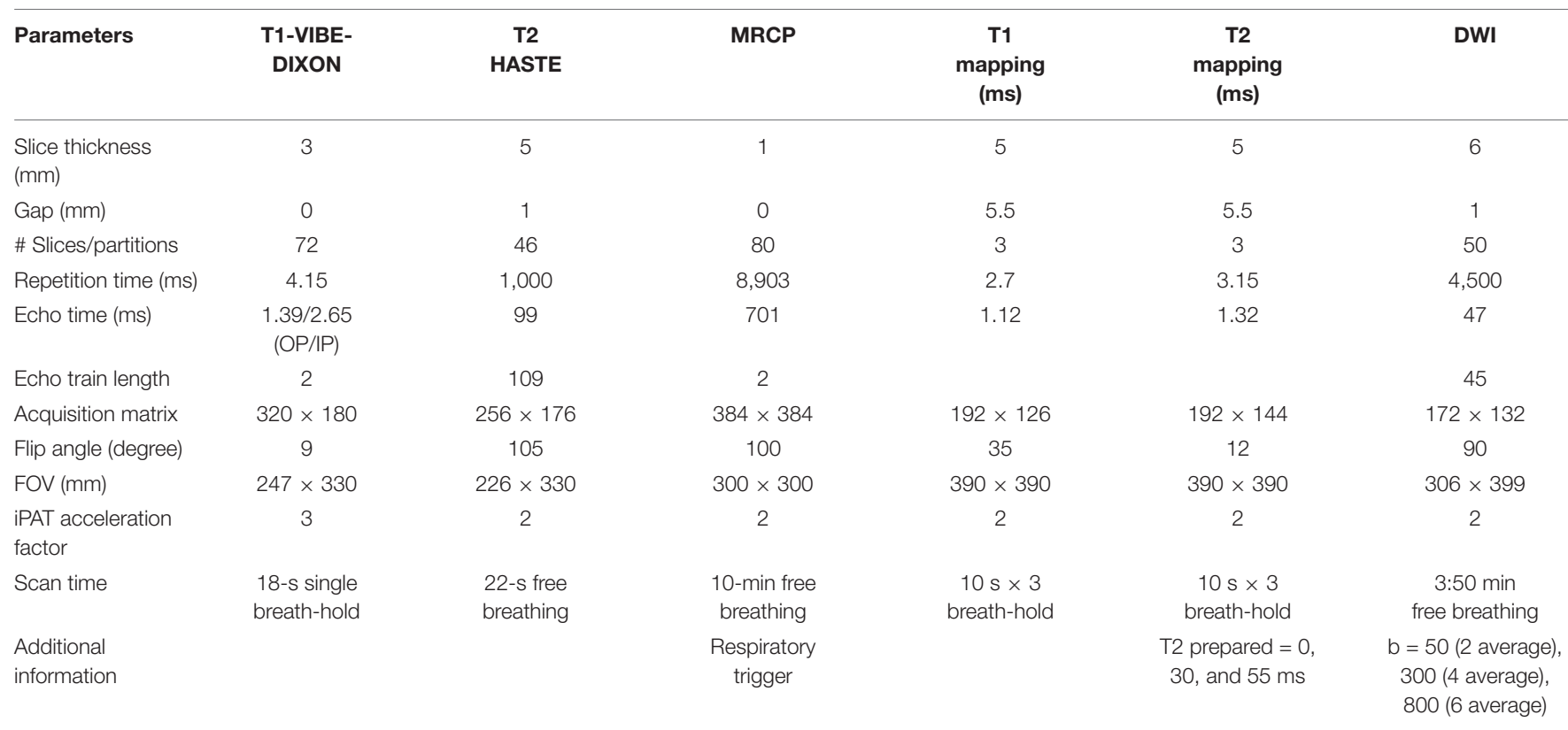


were acquired. DWI covered from the dome of the diaphragm to the lower edge of the kidney using the following parameters: $b$-values $=50,400$, and $800 \mathrm{~s} / \mathrm{mm}^{2}, \mathrm{TR}=4,500 \mathrm{~ms} ; \mathrm{TE}=47 \mathrm{~ms}$; flip angle, $12^{\circ}$; FOV, $390 \times 390 \mathrm{~mm}$; matrix, $192 \times 144$; $\mathrm{ETL}=45$; slice thickness $=5 \mathrm{~mm}$; slice gap $=1 \mathrm{~mm}$; iPAT acceleration factor $=2$, number of averages $=2\left(b=50 \mathrm{~s} / \mathrm{mm}^{2}\right)$, $4\left(b=400 \mathrm{~s} / \mathrm{mm}^{2}\right)$, and $6\left(b=800 \mathrm{~s} / \mathrm{mm}^{2}\right)$. The parameters of all sequences are listed in Table $\mathbf{1 .}$

\section{Imaging Analysis}

T1, T2, and ADC maps of a whole pancreas with PDAC, CP, and normal control pancreas were analyzed and measured along the margin of the pancreas by one radiologist with 20 years of experience in abdominal MRI (LW). We used pixel-wise methods to obtain the mean value of the whole pancreas and avoided areas of necrosis and blood vessels. Tumors were localized with reference to other sequences such as T1-VIBE DIXON, T2 HASTE, DWI images, or the previous contrast-enhanced CT, MRI, or PET/CT within 1 week. In addition, the freehand ROIs (regions of interest) were located within the tumor margin avoiding areas of necrosis. The mean values of tumor, upstream and downstream pancreas (Figures 1, 2), CP (Figure 3), normal pancreatic head, body, and tail (Figure 4) were also obtained based on pixel-wise methods of three slices on T1 and T2 mapping and all the slices on the ADC maps. The tumor's size was defined as the largest diameter in axial images according to the RECIST 1.1 criteria.

\section{Statistical Analysis}

All the data were analyzed on SPSS v22.0 (Armonk, NY, United States; IBM Corp) and MedCalc (MedCalc for Windows, version 16.2.0.0, Mariakerke, Belgium). Data from the regions of interest were tabulated in a Microsoft Excel worksheet (Microsoft Corporation, Seattle, WA, United States). Descriptive statistics (mean values and the standard deviation) were obtained for the whole pancreas with PDAC, CP, and normal control pancreas, for PDAC mass, upstream and downstream pancreas, and for the head, body, and tail of the normal control pancreas. ANOVA tests were used to compare quantitative parameters between groups, and post hoc Tukey tests were used for multiple comparison testing within groups. The value of $p<0.05$ was considered significant. ROC curve analysis and logistic regression was performed to evaluate the sensitivity and specificity of single parameters and each combination of multiple

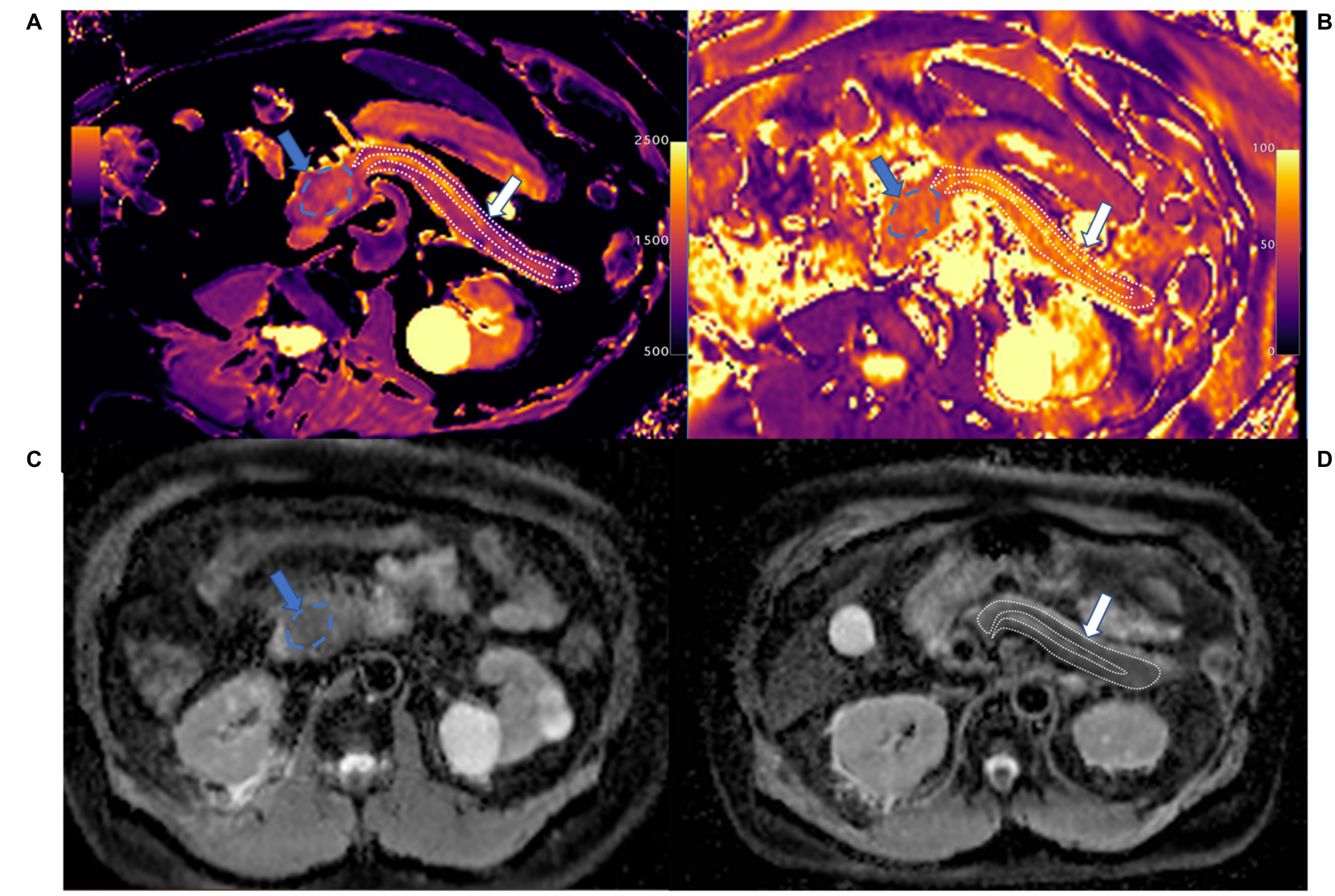

FIGURE 1 | T1 mapping (A), T2 mapping (B), and apparent diffusion coefficient (ADC) maps (C,D) of pancreatic ductal adenocarcinoma (PDAC; blue arrow and area) and upstream pancreas (white arrow and area). PDAC shows higher T1 (1,646.7 $\pm 96.1 \mathrm{~ms}$ ) and T2 (65.1 $\pm 9.4 \mathrm{~ms})$ values compared with upstream pancreas, and slightly higher ADC values (1.326 $\left.\pm 0.098 \mathrm{~mm}^{2} / \mathrm{s}\right)$ compared with upstream pancreas on ADC maps. Upstream pancreas shows homogeneous lower T1 (1,405.2 $\pm 149.9 \mathrm{~ms})$, iso-T2 (61.5 $\pm 9.2 \mathrm{~ms})$, and lower ADC values (1.095 $\left.\pm 0.261 \mathrm{~mm}{ }^{2} / \mathrm{s}\right)$. The MPD shows marked dilation and high T1 and ADC values. 


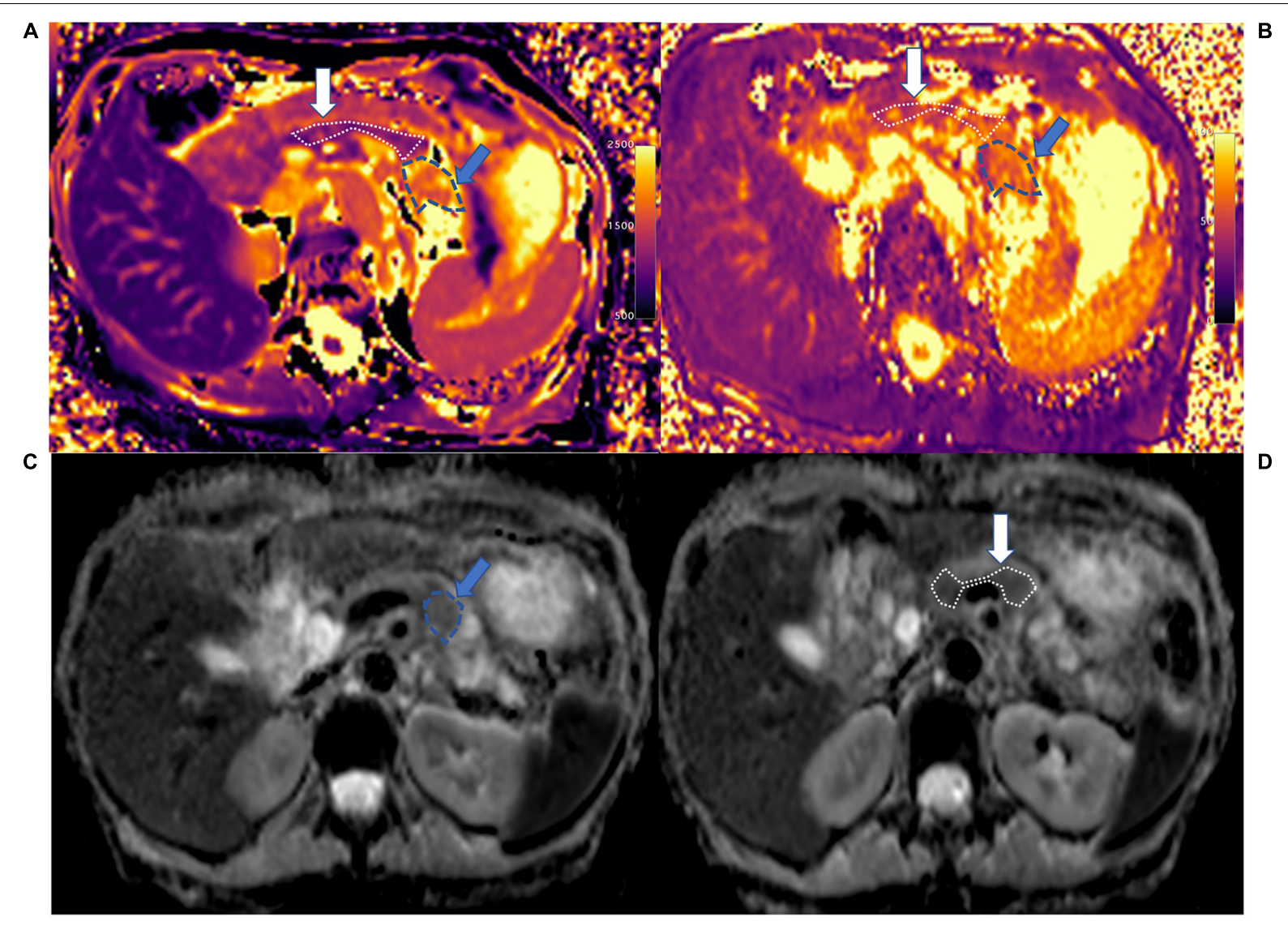

FIGURE 2 | T1 mapping (A), T2 mapping (B), and ADC maps (C,D) of PDAC (blue arrow and area) and downstream pancreas (white arrow). PDAC shows higher T1 $(1,867.3 \pm 163.7 \mathrm{~ms})$, T2 $(69.5 \pm 6.0 \mathrm{~ms})$, and ADC values $\left(1.857 \pm 0.231 \mathrm{~mm}^{2} / \mathrm{s}\right)$ compared to downstream pancreas. The downstream pancreas shows homogenous T1 $(1,266.4 \pm 106.9 \mathrm{~ms})$, T2 $(62.7 \pm 6.3 \mathrm{~ms})$, and ADC $\left(1.315 \pm 0.173 \mathrm{~mm}^{2} / \mathrm{s}\right)$ values. A clear interface is seen between the tumor and downstream pancreas.

parameters in the prediction of PDAC after chemotherapy, and the AUCs were calculated. The value of multiparametric mapping over single parameter mapping was determined in MedCalc, using the Z-statistic to compare the AUCs of the different ROC curves.

\section{RESULTS}

\section{Demographics}

A total of 53 imaging studies were included (17 patients with PDAC, 7 patients with CP, and 29 volunteers with normal healthy pancreas). Among 17 patients with PDAC, 9 patients were male, and 8 patients were female. The mean age was 65 years old with a range of $46-80$ years.

All patients with PDAC were confirmed by FNA and/or surgical specimen. Six lesions were moderately differentiated adenocarcinomas, three lesions were poorly differentiated adenocarcinomas, and the other seven lesions were read as invasive ductal adenocarcinoma, but the grade was not reported.

Based on expert radiologist review of MR images (LW), nine (52.9\%) PDACs were located in the pancreatic head, three $(17.6 \%)$ in the pancreatic neck, two (11.8\%) PDACs in the pancreatic body, and three (17.6\%) in the pancreatic tail. The median size for all tumors was $3.2 \mathrm{~cm}$, with a range from 1.3 to $6.7 \mathrm{~cm}$.

Among the seven patients (four males and three females) with $\mathrm{CP}$, the mean age was 53 years old with a range of $30-72$ years. The mean width of MPD was $4.0 \mathrm{~mm}$ with a range from 1.6 to $6.7 \mathrm{~mm}$.

Among the 29 volunteers with normal healthy pancreas, 13 were male and 16 were female. The mean age was 50 years old with a range of $20-72$ years.

\section{Characteristics, Quantification, and Comparison of T1, T2, and ADC Maps}

For the whole pancreas with PDAC, CP, and normal control pancreas, T1, T2, and ADC values are listed in Table 2 (Figure 5). Significant differences were found between the three groups $(p<0.001)$. The whole pancreas with PDAC showed the highest T1 value compared with the $\mathrm{CP}$ and normal control pancreas with significant differences found $(p<0.001)$. T1 values for CP were significantly higher than those for the normal pancreas $(p<0.001)$. Significant differences were found when comparing 


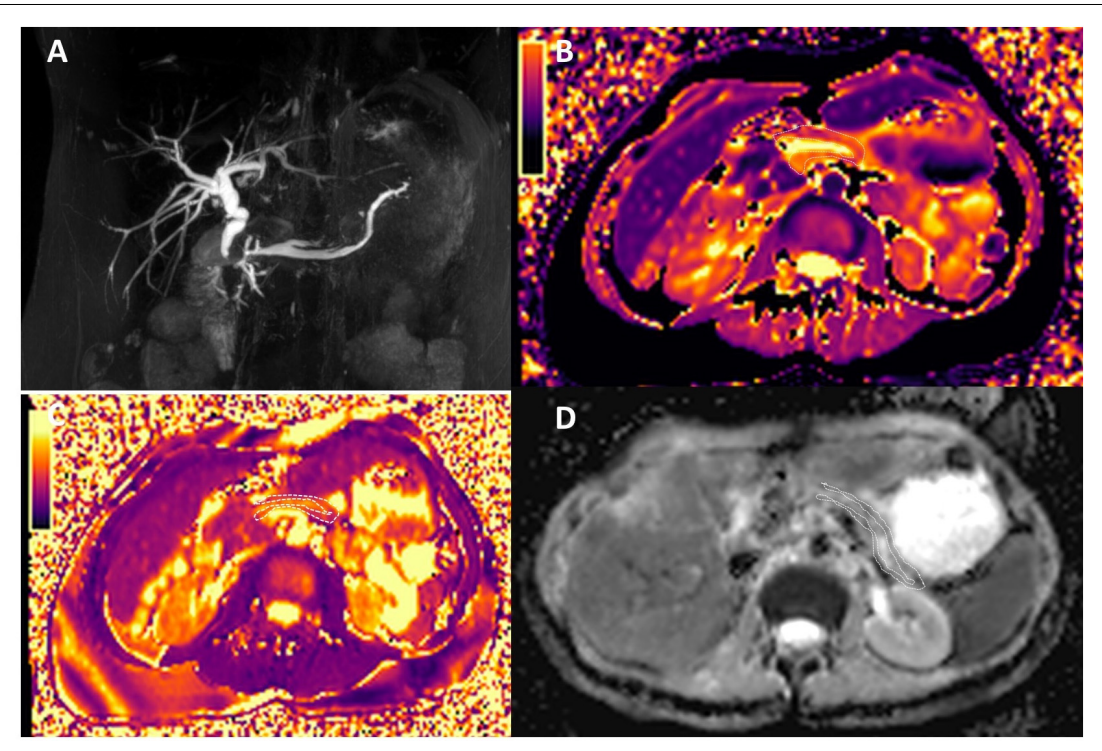

FIGURE 3 | Magnetic resonance cholangiopancreatography (A), T1 mapping (B), T2 mapping (C), and ADC maps (D) of chronic pancreatitis (CP). The patient had the history of $\mathrm{CP}$, and the amylase in the blood elevated. MRCP shows dilatation of the major pancreatic duct (MPD) and the branch pancreatic duct; the width of MPD was $4.5 \mathrm{~mm}$. The T1, T2, and ADC of CP were $1,320 \pm 220.9 \mathrm{~ms}, 57.0 \pm 9.6 \mathrm{~ms}$, and $1.320 \pm 0.162 \mathrm{~mm}^{2} / \mathrm{s}$, respectively.

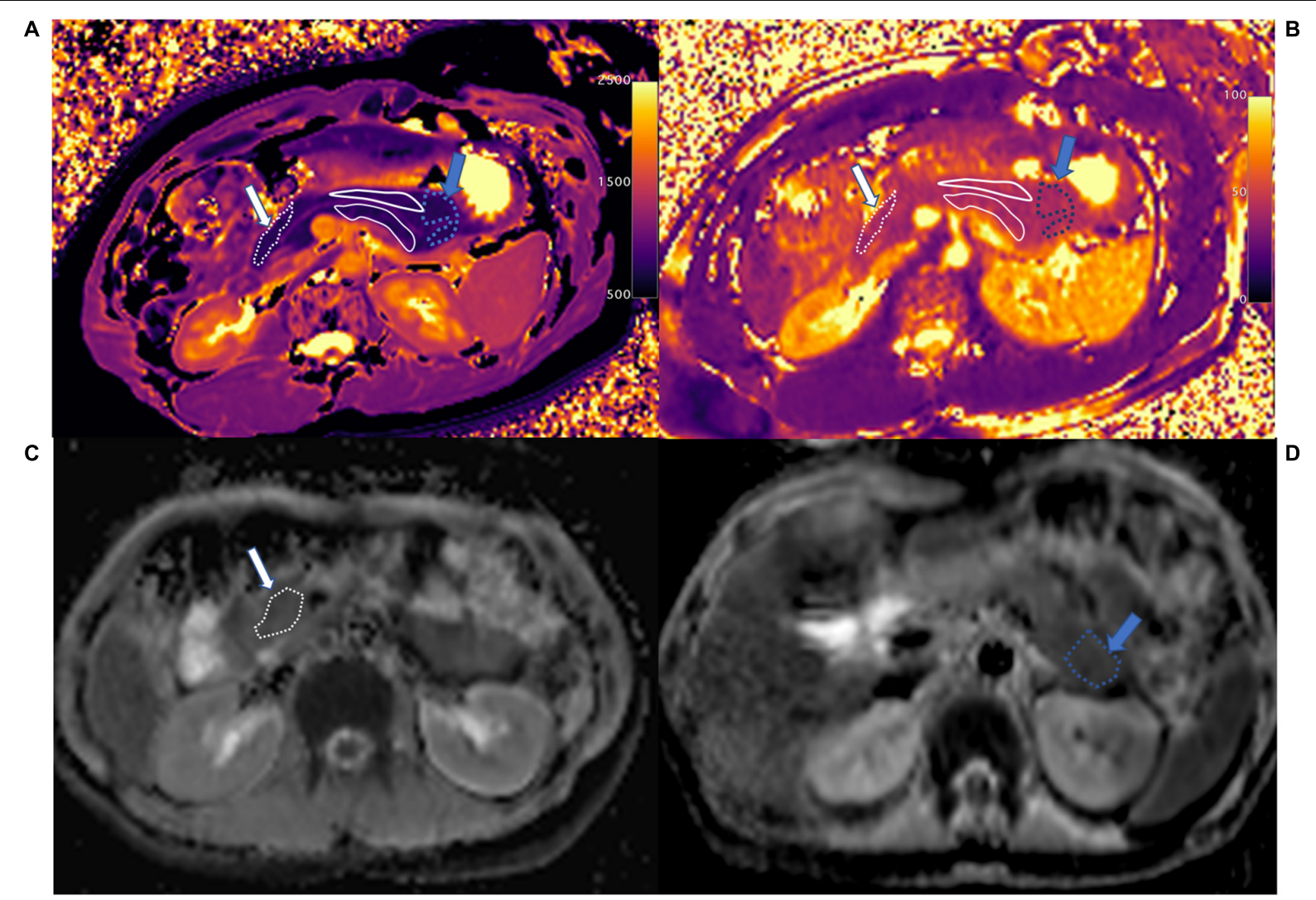

FIGURE 4 | T1 mapping (A), T2 mapping (B), and ADC maps (C,D) of normal control pancreatic head (white arrow and area), body (white solid line area), and tail (blue arrow and area). The whole pancreas had the homogenous T1 (head: $798.2 \pm 80.5 \mathrm{~ms}$, body: $832.0 \pm 83.5 \mathrm{~ms}$, and tail: $835.2 \pm 49.7 \mathrm{~ms}$ ), T2 (head: $45.6 \pm 6.5 \mathrm{~ms}$, body: $44.4 \pm 4.0 \mathrm{~ms}$, and tail: $45.8 \pm 2.2 \mathrm{~ms}$ ), and ADC (head: $1.206 \pm 0.069 \mathrm{~mm}^{2} / \mathrm{s}$, body: $1.141 \pm 0.183 \mathrm{~mm}^{2} / \mathrm{s}$, and tail: $1.061 \pm 0.108 \mathrm{~mm} / \mathrm{s}$ ) values in the head, body, and tail. The main pancreatic duct (MPD) shows clearly on T1 mapping, which differs from the pancreatic head and tail. 
TABLE 2 | T1, T2, and ADC values of the whole pancreas with PDAC, chronic pancreatitis (CP), and normal control pancreas.

\begin{tabular}{|c|c|c|c|}
\hline & $\mathrm{T} 1$ value $($ mean $\pm \mathrm{SD})(\mathrm{ms})$ & $\mathrm{T} 2$ value $($ mean $\pm \mathrm{SD})(\mathrm{ms})$ & ADC value (mean $\pm S D)\left(\times 10^{-3} \mathrm{~mm}^{2} / \mathrm{s}\right)$ \\
\hline Chronic pancreatitis & $1,324.0 \pm 222.9$ & $57.7 \pm 10.7$ & $1.345 \pm 0.174$ \\
\hline$p$-Value ${ }^{*}$ & $<0.001$ & $<0.001$ & $<0.001$ \\
\hline
\end{tabular}

${ }^{*} p$-Value is the difference between three groups.

A

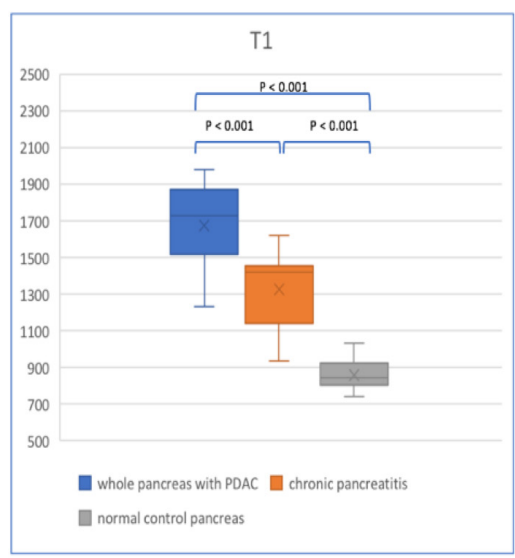

B

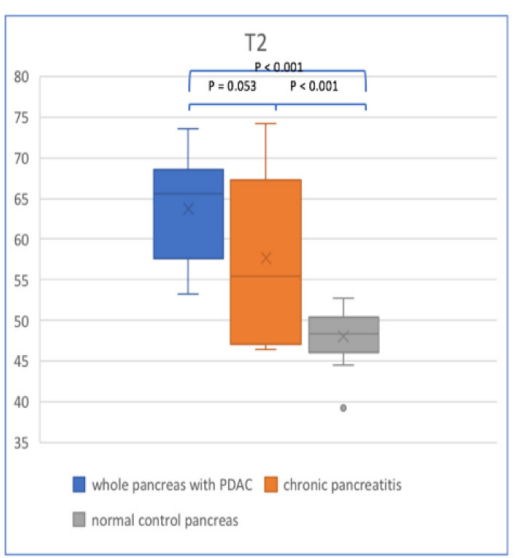

C

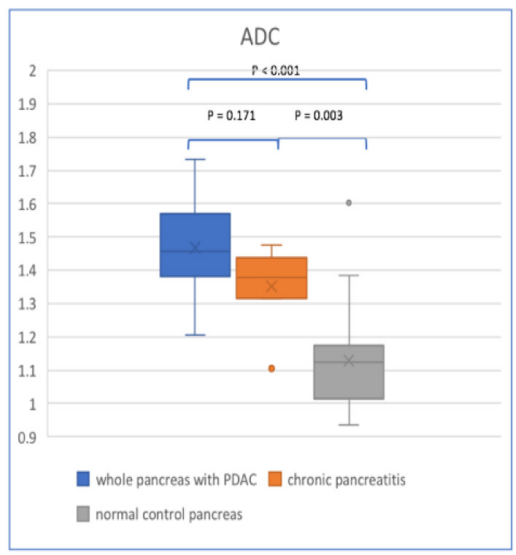

FIGURE 5 | Box plots show T1 (A), T2 (B), and ADC (C) values of the whole pancreas with PDAC, CP, and normal control pancreas. $p$-values are listed on the upper box according to one-way analysis of variance (ANOVA).

TABLE 3 | T1, T2, and ADC values of the PDAC, downstream, and upstream.

$\mathrm{T} 1$ value $($ mean $\pm \mathrm{SD})(\mathrm{ms})$

\section{PDAC}

Downstream pancreas

Upstream pancreas

$p$-Value*

$\begin{aligned} 1,816.5 & \pm 208.5 \\ 1,133.1 & \pm 225.7 \\ 1,598.5 & \pm 292.0 \\ <0.001 & \end{aligned}$

$\mathrm{T} 2$ value (mean $\pm \mathrm{SD})(\mathrm{ms})$

$64.9 \pm 7.6$
$54.9 \pm 6.9$
$61.0 \pm 9.2$
0.037

$A D C$ value (mean $\pm S D)\left(\times 10^{-3} \mathrm{~mm}^{2} / \mathrm{s}\right)$

$$
\begin{aligned}
& 1.525 \pm 0.243 \\
& 1.231 \pm 0.168 \\
& 1.355 \pm 0.194 \\
& 0.003
\end{aligned}
$$

${ }^{*} p$-Value is the difference between three groups.

A

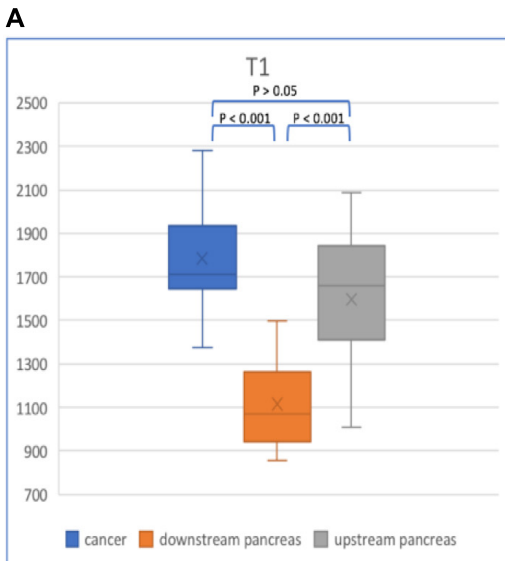

$\square$ cancer $\square$ downstream pancreas $\square$ upstream pancreas
B

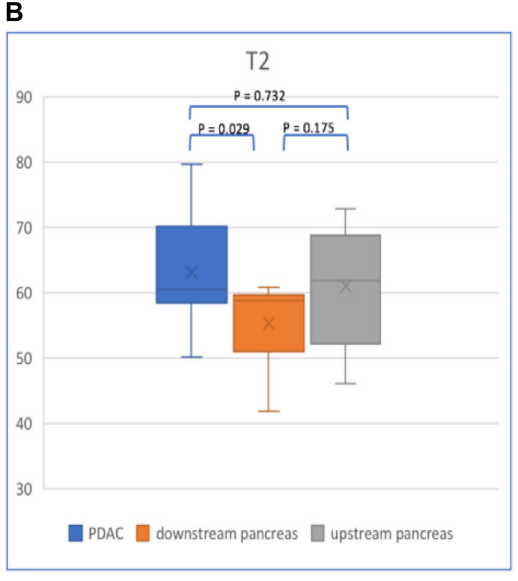

C

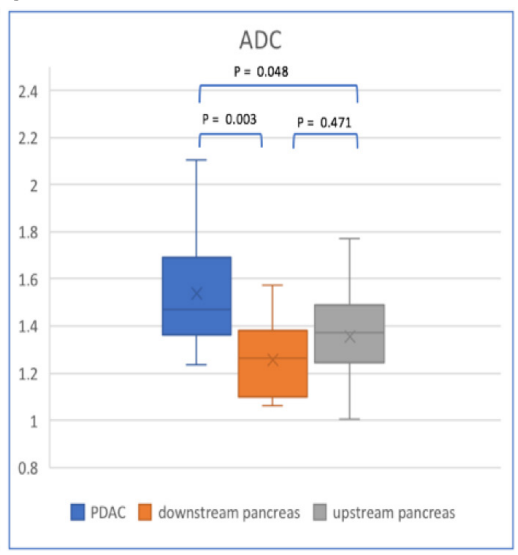

FIGURE 6 | Box plots show T1 (A), T2 (B), and ADC (C) values of PDAC, downstream and upstream pancreas. p-values are listed on the upper box according to one-way ANOVA. 
T2 and ADC values of the whole pancreas with PDAC to normal pancreas $(p<0.001)$. In addition, T2 and ADC values of CP had significant differences compared to the normal pancreas $(p<0.001$ and $p=0.003)$. However, no significant differences were found in the whole pancreas in T2 and ADC between PDAC and $\mathrm{CP}(p=0.053$ and $p=0.171)$.

In patients with PDAC, the $\mathrm{T} 1, \mathrm{~T} 2$, and $\mathrm{ADC}$ values of mass, upstream and downstream pancreas are listed in Table 3 (Figure 6). The PDAC mass had the highest T1, $\mathrm{T} 2$, and ADC values compared with those of the upstream and downstream pancreas. The T1 values showed a significant difference $(p<0.001)$ when comparing the PDAC tumor with the downstream pancreas. The downstream pancreas showed statistically significant differences with the upstream pancreas $(p<0.001)$. No significant differences were found between the PDAC and upstream pancreas $(p>0.05)$. The T2 values were statistically significant $(p=0.029)$ between the PDAC and downstream pancreas, but no significant differences were observed between the PDAC and upstream pancreas $(p=0.732)$, and between the upstream and downstream pancreas $(p=0.175)$. The ADC values were significantly different when comparing PDAC with the upstream $(p=0.048)$ and downstream pancreas $(p=0.003)$. However, no significant difference was found between the upstream and downstream pancreas $(p=0.471)$.

In normal pancreas, the head, body, and tail showed relatively homogenous appearances (Figure 7). The T1, T2, and ADC values are listed in Table 4. No significant differences in T1, T2, and ADC values were found between the normal pancreatic head, body, and tail $(p>0.05)$.

Comparisons of the parameters between PDAC mass and non-tumor parts of the pancreas in patients with PDAC, CP, and normal pancreas were performed (Table 5). T1 values were significantly different $(p<0.001)$ when comparing PDAC with non-tumor parts of the pancreas, $C P$, and normal pancreas. T2 and ADC value differences were statistically significant $(p<0.05)$ when comparing PDAC with non-tumor pancreas and normal pancreas. ADC values also shows statistically significant differences $(p<0.05)$ when comparing non-tumor pancreas and
$\mathrm{CP}$ with normal pancreas. However, no significant differences between $\mathrm{CP}$ and non-tumor pancreas were found in T1, T2, and ADC values $(p>0.05)$. T2 and ADC values also showed no significant differences between PDAC and CP $(p>0.05)$.

\section{Differentiation of PDAC Mass From Non-tumor Pancreas}

Evaluation by ROC curves and the AUCs for each parameter and combinations of parameters was performed (Figure 8). Using single parameters, T1 values yielded the greatest AUC (95\% CI) $[0.901(0.874-0.928)]$ compared to T2 [0.552 (0.507-0.598)] and ADC $[0.712(0.672-0.752)]$. Based on the ROC analysis, the cutoff values of $\mathrm{T} 1, \mathrm{~T} 2$, and $\mathrm{ADC}$ for the differentiation of PDAC mass undergoing chemotherapy from non-tumor pancreas were $1,494.0,59.3 \mathrm{~ms}$, and $1.201 \times 10^{-3} \mathrm{~mm}^{2} / \mathrm{s}$, respectively. The sensitivity and specificity were for T1 (96.55 and $78.39 \%)$, T2 (87.77 and $31.61 \%)$, and ADC values (91.60 and $47.74 \%$ ) were calculated. When combining two parameters, the highest AUC (95\% CI) was obtained when combining T1 and ADC [0.934 (0.914-0.953)] compared to a combination of $\mathrm{T} 1$ and $\mathrm{T} 2$ [0.913 (0.887-0.938)], or a combination of T2 and ADC [0.725 (0.686-0.763)]. When T1, T2, and ADC were combined, the AUC (95\% CI) was $0.937(0.918-0.956)$ with a sensitivity of $91.54 \%$ and a specificity of $85.81 \%$, and this was significantly higher than that using any single parameter $(p<0.001)$. There was no statistical difference between a combination of T1, T2, and ADC vs. T1 and ADC $(p=0.158)$. The sensitivity and specificity of each curve is listed in Table 6.

\section{DISCUSSION}

In this study, significant differences were found when defining the tissue characteristics of the whole pancreas with PDAC, $\mathrm{CP}$, and normal control pancreas, and comparing PDAC mass, upstream and downstream pancreas using T1, T2, and ADC values. The differences between the measured T1, T2, and

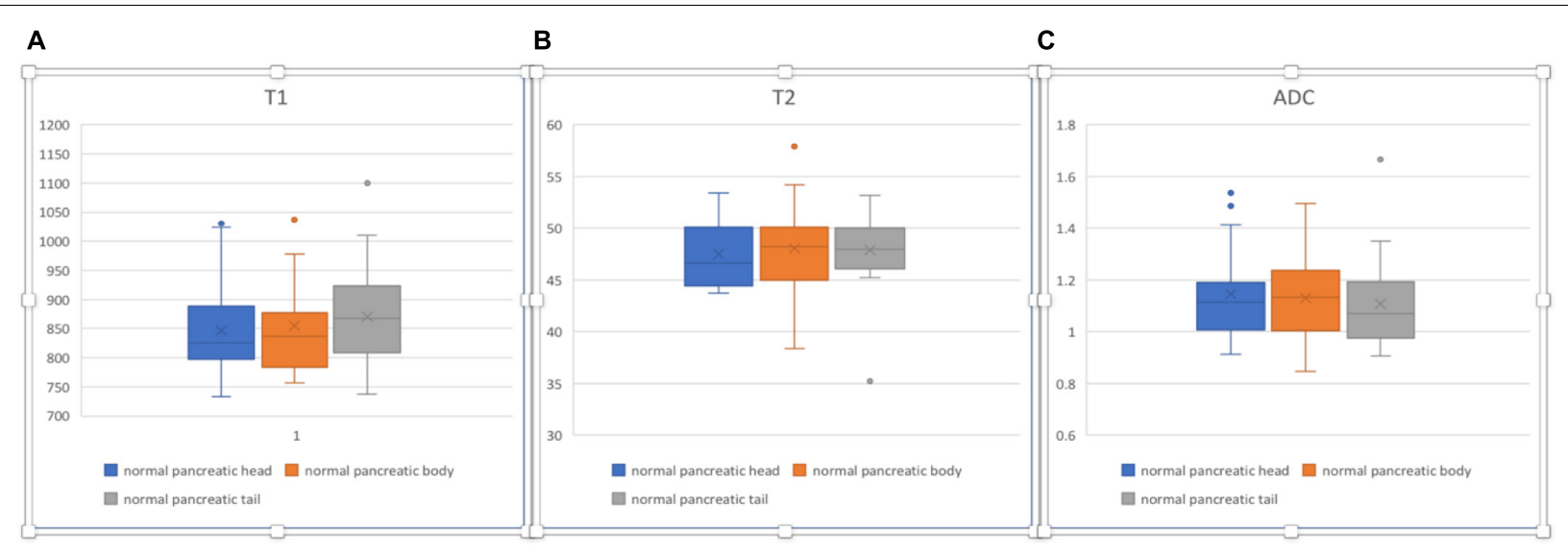

FIGURE 7 | Box plots sho2W T1 (A), T2 (B), and ADC (C) values of normal pancreatic head, body, and tail. No significant differences were found according to one-way ANOVA $(p>0.05)$. 


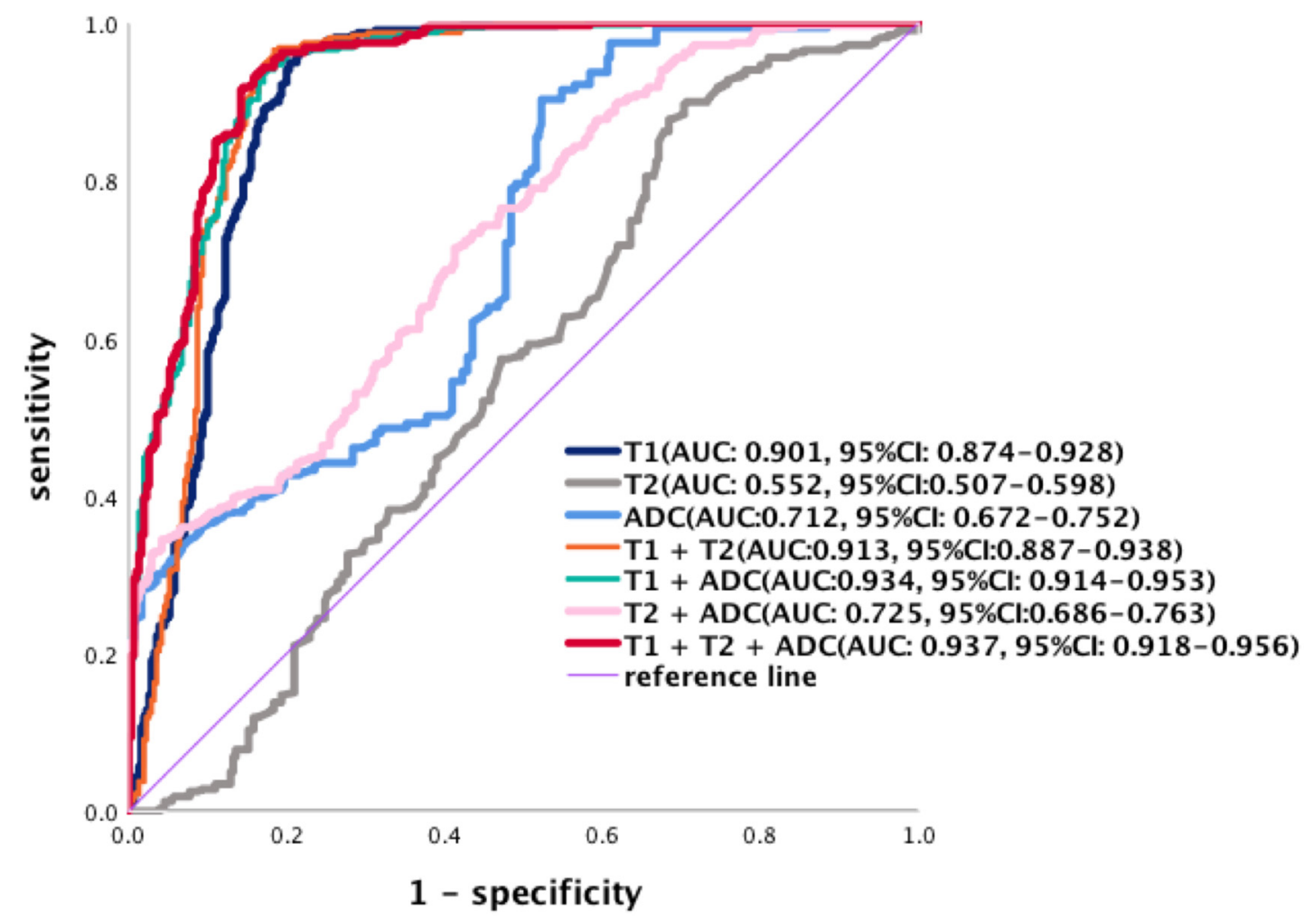

FIGURE 8 | Receiver operating characteristic curves of the diagnostic performance of $\mathrm{T} 1$, $\mathrm{T} 2$, and ADC values and the different combinations of parameters in the differentiation of PDAC. The AUCs (95\% Cl) are T1 [0.901 (0.874-0.928)], T2 [0.552 (0.507-0.598)], and ADC values [0.712 (0.672-0.752)]. Combination of two parameters, the AUCs $(95 \% \mathrm{Cl})$ of T1 + ADC [0.934 (0.914-0.953)], T1 + T2 [0.913 (0.887-0.938)], and T2 + ADC [0.725 (0.686-0.763)]. The AUCs (95\% Cl) for the combination of T1, T2, and ADC are [0.937 (0.918-0.956)].

TABLE 4 | T1, T2, and ADC values of normal pancreatic head, body, and tail.

\begin{tabular}{|c|c|c|c|}
\hline & $\mathrm{T} 1$ value $($ mean $\pm \mathrm{SD})(\mathrm{ms})$ & $\mathrm{T} 2$ value $($ mean $\pm \mathrm{SD})(\mathrm{ms})$ & ADC value (mean $\pm S D)\left(\times 10^{-3} \mathrm{~mm}^{2} / \mathrm{s}\right)$ \\
\hline Normal pancreatic body & $854.6 \pm 85.6$ & $48.1 \pm 4.2$ & $1.129 \pm 0.172$ \\
\hline$p$-Value ${ }^{*}$ & 0.563 & 0.832 & 0.802 \\
\hline
\end{tabular}

${ }^{*} p$-Value is the difference between three groups.

TABLE 5 | p-Values of one-way analysis of variance (ANOVA) in multiple comparisons of PDAC, non-tumor pancreas, CP, and normal control pancreas.

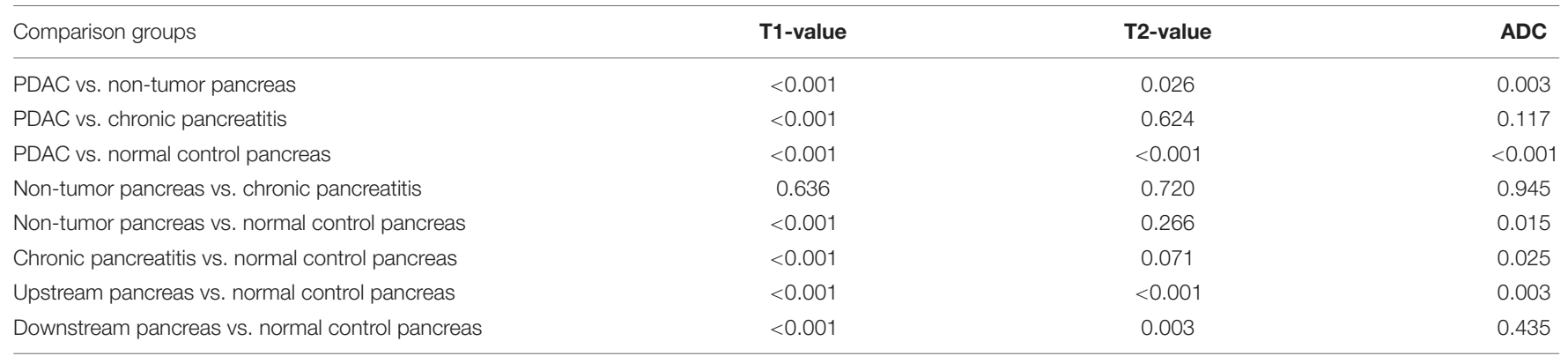

$\mathrm{ADC}$ values in whole pancreas with $\mathrm{PDAC}, \mathrm{CP}$, and normal pancreas may reflect the development of $\mathrm{CP}$ to PDAC in some extent and may provide a new follow-up method of
CP. The difference between non-tumor pancreas, especially upstream pancreas and downstream pancreas, and in normal control pancreas was pathologically proven to correlate with 
TABLE 6 | Sensitivity and specificity of each single parameter and combinations of multi-parameters.

\begin{tabular}{lcc}
\hline & Sensitivity (\%) & Specificity (\%) \\
\hline T1 & 96.55 & 78.39 \\
$\mathrm{~T} 2$ & 87.77 & 31.61 \\
$\mathrm{ADC}$ & 91.60 & 47.74 \\
$\mathrm{~T} 1+\mathrm{T} 2$ & 96.55 & 81.61 \\
$\mathrm{~T} 1+\mathrm{ADC}$ & 93.73 & 82.90 \\
$\mathrm{~T} 2+\mathrm{ADC}$ & 34.5 & 95.8 \\
$\mathrm{~T} 1+\mathrm{T} 2+\mathrm{ADC}$ & 91.54 & 85.81 \\
\hline
\end{tabular}

the inflammatory pathological change, which plays an important role on the tumor response of chemotherapy, recurrence, and 5-year survival rate (Hayano et al., 2016). Furthermore, we found that a combination of $\mathrm{T} 1, \mathrm{~T} 2$, and $\mathrm{ADC}$ values was superior in characterizing tissue properties when compared to any single parameter.

Normal healthy pancreas has the lowest T1, T2, and ADC values compared to the PDAC group and CP group. No significant differences were found between the pancreatic head, body, and tail $(p>0.05)$. Normal healthy pancreas has a short T1 value compared with other abdominal organs due to the presence of a high amount of acinar protein and rough endoplasmic reticulum in the pancreatic cells (Tirkes et al., 2017; Noda et al., 2019). In our study, the mean ADC value showed a slight trend of reduction from head to tail (head $1.146 \times 10^{-3} \mathrm{~mm}^{2} / \mathrm{s}$, body $1.129 \times 10^{-3} \mathrm{~mm}^{2} / \mathrm{s}$, and tail $1.108 \times 10^{-3} \mathrm{~mm}^{2} / \mathrm{s}$ ) due to the heterogeneity of pancreatic tissue composition, and this is consistent with the prior report (Schoennagel et al., 2011).

Chronic pancreatitis results in irreversible pancreatic structural damage, and the pathological changes include pancreatic calcification, chronic inflammation, and fibrosis, which correlate with an increase in $\mathrm{T} 1$ signal intensity in pancreatic parenchyma (Yoon et al., 2016). An increased T1 value was demonstrated with progressive disease (normal controls $865 \pm 220 \mathrm{~ms}$ vs. mild CP 1,075 $\pm 221 \mathrm{~ms}$ vs. severe CP $1,350 \pm 139 \mathrm{~ms}, p<0.0001$ ) (Wang et al., 2018) in a previous study. In our study, the T1 value in CP was markedly higher than that of the normal control pancreas $(p<0.001)$ and lower than that of the whole pancreas with PDAC $(p<0.001)$. CP also has longer T2 and higher ADC than those of the normal control pancreas, which may be related with edema, fibrosis, gland atrophy, and fat infiltration.

\section{Key points}

- Chronic pancreatitis (CP) is associated with a marked increase in the risk of pancreatic ductal adenocarcinoma (PDAC). The differentiation of PDAC and $\mathrm{CP}$ is an important topic.

- Using multi-dimensional MRI methods we found significant differences in T1, $\mathrm{T} 2$ and $\mathrm{ADC}$ values in tissue characteristics of the whole pancreas between PDAC, $\mathrm{CP}$ and normal control pancreas.

- Combining T1, T2 and ADC values provided greatest discrimination between PDAC, CP and normal control pancreas.

FIGURE 9 | The key points about this article.
The T1, T2, and ADC values of the whole pancreas with PDAC were highest compared with the other two groups for the malignant tumor, fibrosis, and COP in the upstream pancreas and TACP around the tumor. The differences in T1 values were statistically significant within the whole pancreas with PDAC, $\mathrm{CP}$, and normal control pancreas $(p<0.001)$. However, T2 and ADC values showed no significant differences between the whole pancreas with PDAC and CP for the pathological changes of $\mathrm{CP}$ and upstream pancreas.

Pancreatic ductal adenocarcinoma tends to obstruct MPD, which results in COP, fibrosis, and atrophy of the upstream pancreas (Balkwill and Mantovani, 2001; Coussens and Werb, 2002; Mantovani et al., 2008). COP may be caused by the tumor obstruction of MPD, which leads to increased intraductal pressure and damage to the duct membrane or rupture of the secondary ducts resulting in interstitial extravasation of the activated pancreatic enzymes, the recurrence of inflammation, and interstitial damage results in fibrosis hyperplasia and CP. Another hypothesis is that tumor cells may secrete plasminogen-activating enzymes, which may, in turn, activate trypsinogen-inducing auto-digestion. In some patients, tumor can arise from preexisting CP (Leal and Liby, 2018). On the other hand, the downstream pancreas may be less influenced than the upstream pancreas.

Significant differences $(p<0.05)$ when comparing nontumor pancreas with PDAC mass, upstream pancreas with downstream pancreas, and downstream pancreas with normal control pancreas can be interpreted by COP or TACP (Imamura et al., 1995). TACP was found in the adjacent parenchyma of the PDAC mass. In addition, this can be used to interpret why $\mathrm{T} 1$ and $\mathrm{T} 2$ values in the downstream pancreas showed significant differences when compared with the normal control pancreas $(p<0.05)$. The results also proved that the inflammatory changes and fibrosis in the upstream pancreas are markedly more severe than the downstream pancreas (Bali et al., 2011).

Receiver operator characteristic analysis showed that for the single parameters, the $\mathrm{T} 1$ value is the most accurate in differentiating the tumor and non-tumor area compared with that of the T2 value and ADC value $(p<0.001)$. The T2 and $\mathrm{ADC}$ values have some limitations when a large amount of fibrosis and the change in tumor cellular density are present in the tumor and upstream pancreas. In our study, we found that if three parameters were combined, the AUC was statistically significantly higher compared with any single parameter and two combined parameters $(p<0.05)$ with the exception of the combined T1 and ADC values $(p=0.158)$. Therefore, it appears that the T1 value is critical in tissue characterization, while the $\mathrm{ADC}$ value can provide additional helpful information but has lower sensitivity and specificity than the T1 value. Based on our data, the T2 value only provides minimal information in tissue characterization.

Our study has some limitations. First, the relatively small numbers of patients may affect the statistical outcomes. These findings will need to be validated in larger studies. Second, patients were not sub-grouped according to the grade of cancer; 
therefore, the T1, T2, and ADC values may show wide variability. Subgrouping of patients was not possible due to the small pilot nature of this study. Third, most patients with PDAC underwent chemotherapy at the time of study. This may have altered the tumor characteristics. We found from our research group experience that patients who were treatment naïve are difficult to recruit. Fourth, the ROIs were obtained according to the previous imaging data and other non-contrast MR images, and there was an interval between the MR scan and surgery. The tumor margin could not be validated immediately, so we selected a relatively smaller ROI within the tumor, avoiding the necrosis area. Fifth, T1 and T2 mapping only included three slices because we used the T1 MOLLI sequence. This would not reflect the global features of the tumor. Future studies should recruit patients prior to initiation of treatment and over the course of the treatment so that there are independent predictors of prognosis in the responder and non-responder. Finally, the T1, T2, and ADC maps had different resolutions at the time of image acquisition, which required retrospective calibration to keep the pixel numbers consistent. In future studies, we will keep the same T1, T2, and DWI image resolutions and continuous or three-dimensional T1 and T2 mapping sequences in imaging acquisition to decrease the bias. For the multiparametric mapping, which can demonstrate the differences of PDAC mass undergoing chemotherapy, upstream and downstream pancreas, we believe that it can be used as a biomarker to predict tumor or longitudinal follow-up after chemotherapy.

\section{CONCLUSION}

In conclusion, multiparametric mapping is feasible for the evaluation of the differences between PDAC, CP, and normal pancreas tissue. The combination of multiple parameters of $\mathrm{T} 1, \mathrm{~T} 2$, and ADC provides a higher accuracy compared to the result with any single parameter in tissue characterization of the pancreas (the main points of this article are listed in Figure 9).

\section{REFERENCES}

American Cancer Society (2018). Cancer Facts \& Figures 2018 American Cancer Society. New York, NY: American Cancer Society.

Apprich, S., Mamisch, T. C., Welsch, G. H., Stelzeneder, D., Albers, C., Totzke, U., et al. (2012). Quantitative T2 mapping of the patella at 3.0T is sensitive to early cartilage degeneration, but also to loading of the knee. Eur. J. Radiol. 81:e00438-43. doi: 10.1016/j.ejrad.2011.03.069

Attiyeh, M. A., Chakraborty, J., Mcintyre, C. A., Kappagantula, R., Chou, Y., Askan, G., et al. (2019). CT radiomics associations with genotype and stromal content in pancreatic ductal adenocarcinoma. Abdom. Radiol. 44, 3148-3157. doi: 10.1007/s00261-019-02112-1

Bali, M. A., Metens, T., Denolin, V., Delhaye, M., Demetter, P., Closset, J., et al. (2011). Tumoral and nontumoral pancreas: correlation between quantitative dynamic contrast-enhanced MR imaging and histopathologic parameters. Radiology 261, 456-466. doi: 10.1148/radiol.11103515

\section{DATA AVAILABILITY STATEMENT}

All datasets generated for this study are included in the article/supplementary material.

\section{ETHICS STATEMENT}

The studies involving human participants were reviewed and approved by the Cedars-Sinai Institutional Review Board. The patients/participants provided their written informed consent to participate in this study. Written informed consent was obtained from the individual(s) for the publication of any potentially identifiable images or data included in this article.

\section{AUTHOR CONTRIBUTIONS}

LW, DL, and SP conceived and designed the study. LW and NW analyzed and interpreted the data. NW, ZD, ZZ, and $\mathrm{AH}$ collected the data. LW and SG wrote the manuscript. SG, ZF, TJ, AC, FH, SL, and AW critically revised the manuscript. DL and SP approved the final version of the manuscript. LW and YX contributed to statistical analysis.

\section{FUNDING}

This research was supported by the Biomedical Imaging Research Institute and Division of Digestive and Liver Diseases of Cedars-Sinai Medical Center, and National Institutes of Health US U01 DK108314-05.

\section{ACKNOWLEDGMENTS}

The research group thanks all the patients and normal volunteers who participated in this study. In addition, we thank Xiaoming Bi, Edward Gill, Joselyn Ricafrente, and Laura Smith who assisted with this study and the MR scan.

Balkwill, F., and Mantovani, A. (2001). Inflammation and cancer: back to Virchow? Lancet 357, 539-545. doi: 10.1016/s0140-6736(00)04046-0

Banerjee, R., Pavlides, M., Tunnicliffe, E. M., Piechnik, S. K., Sarania, N., Philips, R., et al. (2014). Multiparametric magnetic resonance for the non-invasive diagnosis of liver disease. J. Hepatol. 60, 69-77. doi: 10.1016/j.jhep.2013.09.002

Blystad, I., Warntjes, J. B. M., Smedby, O., Lundberg, P., Larsson, E. M., and Tisell, A. (2017). Quantitative MRI for analysis of peritumoral edema in malignant gliomas. PLoS One 12:e0177135. doi: 10.1371/journal.pone.0177135

Cassinotto, C., Feldis, M., Vergniol, J., Mouries, A., Cochet, H., Lapuyade, B., et al. (2015). MR relaxometry in chronic liver diseases: comparison of T1 mapping, T2 mapping, and diffusion-weighted imaging for assessing cirrhosis diagnosis and severity. Eur. J. Radiol. 84, 1459-1465. doi: 10.1016/j.ejrad.2015.05.019

Chen, C., Chen, J., Xia, C., Huang, Z., and Song, B. (2017). T1 mapping combined with Gd-EOB-DTPA-enhanced magnetic resonance imaging in predicting the pathologic grading of hepatocellular carcinoma. J. Biol. Regul. Homeostat. Agents 31, 1029-1036. 
Choi, S.-Y., Kim, S. H., Kang, T. W., Song, K. D., Park, H. J., and Choi, Y.-H. (2016). Differentiating mass-forming autoimmune pancreatitis from pancreatic ductal adenocarcinoma on the basis of contrast-enhanced MRI and DWI findings. Am. J. Roentgenol. 206, 291-300. doi: 10.2214/AJR.15. 14974

Cieszanowski, A., Anysz-Grodzicka, A., Szeszkowski, W., Kaczynski, B., Maj, E., Gornicka, B., et al. (2012). Characterization of focal liver lesions using quantitative techniques: comparison of apparent diffusion coefficient values and T2 relaxation times. Eur. Radiol. 22, 2514-2524. doi: 10.1007/s00330-0122519-x

Coussens, L. M., and Werb, Z. (2002). Inflammation and cancer. Nature 420, 860-867.

Freeny, P. C. (1989). Classification of pancreatitis. Radiol. Clin. North Am. 27, 1-3.

Fukukura, Y., Takumi, K., Kamimura, K., Shindo, T., Kumagae, Y., Tateyama, A., et al. (2012). Pancreatic adenocarcinoma: variability of diffusion-weighted MR imaging findings. Radiology 263, 732-740. doi: 10.1148/radiol.12111222

Guo, H., Au, W. Y., Cheung, J. S., Kim, D., Jensen, J. H., Khong, P. L., et al. (2009). Myocardial T2 quantitation in patients with iron overload at 3 Tesla. J. Magn. Reson. Imaging 30, 394-400. doi: 10.1002/jmri.21851

Hao, L., Zeng, X. P., Xin, L., Wang, D., Pan, J., Bi, Y. W., et al. (2017). Incidence of and risk factors for pancreatic cancer in chronic pancreatitis: a cohort of 1656 patients. Dig. Liver Dis. 49, 1249-1256. doi: 10.1016/j.dld.2017.07.001

Hayano, K., Miura, F., Wada, K., Suzuki, K., Takeshita, K., Amano, H., et al. (2016). Diffusion-weighted MR imaging of pancreatic cancer and inflammation: prognostic significance of pancreatic inflammation in pancreatic cancer patients. Pancreatology 16, 121-126. doi: 10.1016/j.pan.2015.10.004

Hecht, E. M., Liu, M. Z., Prince, M. R., Jambawalikar, S., Remotti, H. E., Weisberg, S. W., et al. (2017). Can diffusion-weighted imaging serve as a biomarker of fibrosis in pancreatic adenocarcinoma? J. Magn. Reson. Imaging 46, 393-402. doi: 10.1002/jmri.25581

Ichikawa, T., Erturk, S. M., Motosugi, U., Sou, H., Iino, H., Araki, T., et al. (2007). High-b value diffusion-weighted MRI for detecting pancreatic adenocarcinoma: preliminary results. Am. J. Roentgenol. 188, 409-414. doi: 10.2214/ajr.05.1918

Imamura, T., Iguchi, H., Manabe, T., Ohshio, G., Yoshimura, T., Wang, Z., et al. (1995). Quantitative analysis of collagen and collagen subtypes I, III, and V in human pancreatic cancer, tumor-associated chronic pancreatitis, and alcoholic chronic pancreatitis. Pancreas 11, 357-364. doi: 10.1097/00006676-19951100000007

Kali, A., Choi, E.-Y., Sharif, B., Kim, Y. J., Bi, X., Spottiswoode, B., et al. (2015). Native T1 mapping by 3-T CMR imaging for characterization of chronic myocardial infarctions. JACC 8, 1019-1030. doi: 10.1016/j.jcmg.2015. 04.018

Karamitsos, T. D., Piechnik, S. K., Banypersad, S. M., Fontana, M., Ntusi, N. B., Ferreira, V. M., et al. (2013). Noncontrast T1 mapping for the diagnosis of cardiac amyloidosis. JACC 6, 488-497. doi: 10.1016/j.jcmg.2012.11.013

Kirkegard, J., Mortensen, F. V., and Cronin-Fenton, D. (2017). Chronic pancreatitis and pancreatic cancer risk: a systematic review and meta-analysis. Am. J. Gastroenterol. 112, 1366-1372. doi: 10.1038/ajg.2017.218

Leal, A. S., and Liby, K. T. (2018). Murine models of pancreatitis leading to the development of pancreatic cancer. Curr. Protoc. Pharmacol. 83:e48. doi: 10.1002/cpph.48

Luz, L. P., Al-Haddad, M. A., Sey, M. S., and Dewitt, J. M. (2014). Applications of endoscopic ultrasound in pancreatic cancer. World J. Gastroenterol. 20, 7808-7818. doi: 10.3748/wjg.v20.i24.7808

Mantovani, A., Allavena, P., Sica, A., and Balkwill, F. (2008). Cancer-related inflammation. Nature 454:436. doi: 10.1038/nature07205

Nattermann, C., Goldschmidt, A. J., and Dancygier, H. (1993). Endosonography in chronic pancreatitis-a comparison between endoscopic retrograde pancreatography and endoscopic ultrasonography. Endoscopy 25, 565-570. doi: 10.1055/s-2007-1010406

Noda, Y., Goshima, S., Tsuji, Y., Kajita, K., Kawada, H., Kawai, N., et al. (2019). Correlation of quantitative pancreatic $\mathrm{T} 1$ value and $\mathrm{HbAlc}$ value in subjects with normal and impaired glucose tolerance. J. Magn. Reson. Imaging 49, 711-718. doi: 10.1002/jmri.26242

Park, H. S., Lee, J. M., Choi, H. K., Hong, S. H., Han, J. K., and Choi, B. I. (2009). Preoperative evaluation of pancreatic cancer: comparison of gadolinium-enhanced dynamic MRI with MR cholangiopancreatography versus MDCT. J. Magn. Reson. Imaging 30, 586-595. doi: 10.1002/jmri. 21889

Sahai, A. V., Zimmerman, M., Aabakken, L., Tarnasky, P. R., Cunningham, J. T., Van Velse, A., et al. (1998). Prospective assessment of the ability of endoscopic ultrasound to diagnose, exclude, or establish the severity of chronic pancreatitis found by endoscopic retrograde cholangiopancreatography. Gastrointest. Endosc. 48, 18-25. doi: 10.1016/s0016-5107(98) 70123-3

Schoennagel, B. P., Habermann, C. R., Roesch, M., Hahne, J. D., Arndt, C., Kleibeler, L., et al. (2011). Diffusion-weighted imaging of the healthy pancreas: apparent diffusion coefficient values of the normal head, body, and tail calculated from different sets of b-values. J. Magn. Reson. Imaging 34, 861-865. doi: 10.1002/jmri.22743

Shi, J., and Xue, J. (2019). Inflammation and development of pancreatic ductal adenocarcinoma. Chin. Clin. Oncol. 8:19. doi: 10.21037/cco.2019. 04.02

Siegel, R. L., Miller, K. D., and Jemal, A. (2018). Cancer statistics, 2018. CA Cancer J. Clin. 68, 7-30. doi: 10.3322/caac. 21442

Singh, A., and Faulx, A. L. (2016). Endoscopic evaluation in the workup of pancreatic cancer. Surg. Clin. North Am. 96, 1257-1270. doi: 10.1016/j.suc.2016. 07.006

Tirkes, T., Lin, C., Cui, E., Deng, Y., Territo, P. R., Sandrasegaran, K., et al. (2018). Quantitative MR evaluation of chronic pancreatitis: extracellular volume fraction and MR relaxometry. AJR Am. J. Roentgenol. 210, 533-542. doi: 10. 2214/AJR.17.18606

Tirkes, T., Lin, C., Fogel, E. L., Sherman, S. S., Wang, Q., and Sandrasegaran, K. (2017). T1 mapping for diagnosis of mild chronic pancreatitis. J. Magn. Reson. Imaging 45, 1171-1176. doi: 10.1002/jmri.25428

Vietti Violi, N., Hilbert, T., Bastiaansen, J. A. M., Knebel, J. F., Ledoux, J. B., Stemmer, A., et al. (2019). Patient respiratory-triggered quantitative T2 mapping in the pancreas. J. Magn. Reson. Imaging 50, 410-416. doi: 10.1002/ jmri.26612

Wang, M., Gao, F., Wang, X., Liu, Y., Ji, R., Cang, L., et al. (2018). Magnetic resonance elastography and $\mathrm{T} 1 \mathrm{mapping}$ for early diagnosis and classification of chronic pancreatitis. J. Magn. Reson. Imaging doi: 10.1002/jmri.26008 [Epub ahead of print],

Wang, Y., Chen, Z. E., Nikolaidis, P., Mccarthy, R. J., Merrick, L., Sternick, L. A., et al. (2011). Diffusion-weighted magnetic resonance imaging of pancreatic adenocarcinomas: association with histopathology and tumor grade. J. Magn. Reson. Imaging 33, 136-142. doi: 10.1002/jmri. 22414

Yamamoto, T., Yagi, S., Kinoshita, H., Sakamoto, Y., Okada, K., Uryuhara, K., et al. (2015). Long-term survival after resection of pancreatic cancer: a single-center retrospective analysis. World J. Gastroenterol. 21, 262-268. doi: 10.3748/wjg. v21.i1.262

Yin, T., Peeters, R., Feng, Y., Liu, Y., Yu, J., Dymarkowski, S., et al. (2017). Characterization of a rat orthotopic pancreatic head tumor model using three-dimensional and quantitative multiparametric MRI. NMR Biomed. 30:e3676. doi: 10.1002/nbm. 3676

Yoon, J. H., Lee, J. M., Lee, K. B., Kim, S. W., Kang, M. J., Jang, J. Y., et al. (2016). Pancreatic steatosis and fibrosis: quantitative assessment with preoperative multiparametric MR imaging. Radiology 279, 140-150. doi: 10.1148/radiol. 2015142254

Conflict of Interest: FH was employed by company Siemens Healthineers.

The remaining authors declare that the research was conducted in the absence of any commercial or financial relationships that could be construed as a potential conflict of interest.

Copyright (C) 2020 Wang, Gaddam, Wang, Xie, Deng, Zhou, Fan, Jiang, Christodoulou, Han, Lo, Wachsman, Hendifar, Pandol and Li. This is an open-access article distributed under the terms of the Creative Commons Attribution License (CC BY). The use, distribution or reproduction in other forums is permitted, provided the original author(s) and the copyright owner(s) are credited and that the original publication in this journal is cited, in accordance with accepted academic practice. No use, distribution or reproduction is permitted which does not comply with these terms. 